\title{
Complex processing of goniometric and acoustic signals in the automated control system of the rehabilitation exoskeleton
}

\author{
$N V$ Dorofeev $^{1, *}, A V$ Grecheneva $^{2}$, and $O R$ Kuzichkin $^{2}$ \\ ${ }^{1}$ Vladimir State University, 87 Gor'kogo St, Vladimir, 600000, Russia \\ ${ }^{2}$ Belgorod State University, 85 Pobedy St, Belgorod, 308015, Russia
}

\begin{abstract}
The technique of express-assessment of intervertebral disk changes is described. This technique is based on the automation of goniometric and arthrophonometric measurements to reduce of the trauma in the regulation and using of the rehabilitation exoskeleton. It is characterized by the application of an individual biomechanical model of a human being, built on the basis of an individual n-shaped angular model and a three-dimensional biomechanical model. The using wavelet filtering and neural network algorithms for the detection and classification of pathologies when processing of the goniometric and atrofonometric data. The developed technique is adaptive: the parameters of models and processing units are adjusted to the physiological characteristics of the patient, the neural network coefficients are automatically adjusted during the diagnostics. The processing of acoustic signals is carried out in several bands with interference compensation. Goniometric measurements are built on the basis of an accelerometric phase-metric system. A generalized structure of the influence of biochemical and biophysical factors on the change of intervertebral discs was developed.
\end{abstract}

\section{Introduction}

Throughout the life of a human, his musculoskeletal system undergoes changes related to the growth, the features of a person's way of life, the functioning of various organs and systems. Since the human body is an adaptive single system, a change in the work of certain organs and systems causes changes in the work of others. One of the most important parts of the human musculoskeletal system is the vertebral column - the main part of the axial skeleton. The spine is involved in all movements of the trunk and head and performs not only the function of support, but also serves as a defense of the spinal cord. The mobility and flexibility of the spine is provided by intervertebral discs, which absorb the load on the spine and, together with the synovial fluid, reduce the friction force of the vertebrae during movement. In the case of a decrease in intervertebral discs or a deterioration of their properties, displacement of the vertebral bodies or a change in the shape of the spinal column, compression of the blood vessels and spinal nerves occurs [1].

\footnotetext{
* Corresponding author: dorofeevnv@yandex.ru
} 
As a result, there are various painful sensations and disruption of the functioning of various organs. It should be noted that the displacement of the vertebrae and the change in the shape of the spine can occur not only under the load, but also under pressure on internal organs [2-3], as well as at changes in muscle tone [3].

Thus, constant monitoring of the state of the intervertebral discs, the shape of the spine and the position of the vertebrae in space, the assessment of the maximum possible deviation of the vertebrae (possible motions) under the influence of the load is an important task of diagnosis and rehabilitation of the spine. The purpose of this article is to develop a methodology for rapid assessment of the human intervertebral disc changes in an automated system of regulation of the rehabilitation exoskeleton.

\section{An indirect assessment technique of changes in the intervertebral discs}

Pathological changes of the intervertebral discs depend on the physiological characteristics of a person (genetic features, changes in the properties of cartilaginous tissue, etc.), lifestyle (activity, exercise, nutrition) and diseases (figure 1).

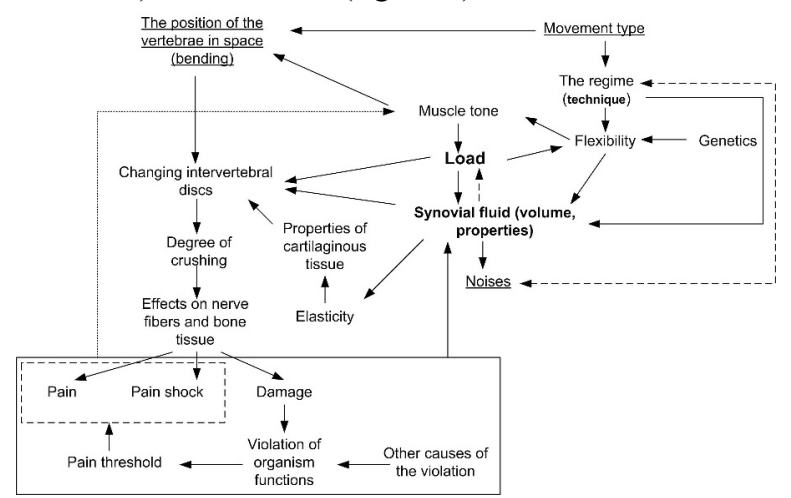

Fig. 1. General structural scheme of the influence of factors on changes in intervertebral discs.

The properties of the cartilaginous tissue of the intervertebral discs, in particular the elasticity, depend on the parameters of the synovial fluid. The simplest mobile unit of the spine is the biotribological segment, which consists of the two vertebrals, intervertebral disc (cartilaginous tissue) and synovial fluid. It is an adaptive cybernetic system, the parameters of which change under the influence of external (load, muscle tone, friction as a result of movement) and internal factors (supply with necessary nutrients) [4 - 6]. It should be noted that the shape and parameters of locomotion (rhythmicity, amplitude, speed, trajectory optimality, sequence, etc.) have a great influence on the elasticity of the cartilaginous tissue. Movement causes a change in the elasticity of the cartilaginous tissue of the intervertebral discs, the flexibility of the spine and can have both a positive (the recovery of the cartilaginous tissue) and a negative (the deformation and a decrease of the intervertebral discs) effects. This means that with the correct distribution of the load (by changing its weight, the tone of the muscles of the whole organism, the type and trajectory of movements) and applying restorative techniques (systematic repetition of movements), the effectiveness of rehabilitation can increase [7-11].

Thus, relying on the dependences obtained by the authors of works [4-11], an estimate of the change in the size of the intervertebral discs and the predictive evaluation of pinching of the spinal nerves and vessels can be obtained by recording the load, the position of the vertebrae in space (spinal forms), the volume and properties of the synovial fluid. The following technique is proposed to solve this problem: 
- an individual biomechanical model is formed for each patient;

- an assessment of the degree of friction of the intervertebral discs is formed on the basis of an individual biomechanical model, recorded movements [7, 8] and load [9-11];

- the predictive assessment of changes in the intervertebral discs is formed on the basis of estimated values of the degree of friction of the intervertebral discs and changes in these estimates over time [7];

- a forecast assessment of the development of disorders of the musculoskeletal system is formed on the basis of predictive estimates of changes in the intervertebral discs, the individual biomechanical model, the degree of activity and the type of movements of the patient. The neural network for data processing (is proposed by the authors of the article in [12]) is also used to forming the forecast assessment.

The resulting estimated and predicted values, as well as the individual biomechanical model, serve as initial data for decision making both in diagnostics and rehabilitation, and in shaping control actions on the executive mechanisms of rehabilitation equipment (exoskeletons, support systems, artificial moving apparatus, etc.).

The individual biomechanical model of the patient is formed on the basis of the individual n-element angular model and the three-dimensional biomechanical model, are described in $[12,13]$, respectively.

The individual n-element angular model is described by a vector $\vec{I}=\left\{z \vec{v}_{1}, \ldots, z v_{i}, \ldots, \vec{z} v_{p}\right\}$ where $z \overrightarrow{v_{i}}=\left\{\vec{\alpha}_{i}, \vec{\beta}_{i}, \vec{\gamma}_{i}\right\}$ is the vector of describing the possible deviations of the link $i$ in space relative to the base point, $\vec{\alpha}_{i}=\left\{\alpha_{1}, \ldots, \alpha_{l}\right\}, \vec{\beta}_{i}=\left\{\beta_{1}, \ldots, \beta_{j}\right\}, \vec{\gamma}_{i}=\left\{\gamma_{1}, \ldots, \gamma_{k}\right\}$ are the possible angular deviations relative to the base point of the link $i$ in the planes $x, y, z$, respectively.

The data for the individual n-element angular model is the goniometric measurements. The data for the formation of a three-dimensional biomechanical model is provided by arthrophonometry, arthrosonometry with ultrasound scanning (to assess the properties and volume of synovial fluid and the degree of friction at the time of measurement, volume of effusion, thickness of the cartilaginous tissue) and other additional information [12, 14].

After the individual biomechanical model of the patient is formed, its further application is carried out on the basis of measurements of dynamic parameters: the goniometric (the motion of the vertebrae and other parts of the body in space) and the arthrophonometric (the articular noise). On the basis of the dependences obtained in [7-11] and the use of adaptive algorithms for data processing [12], an indirect estimate of the degree of loading is possible by the parameters of the synovial fluid and the structural parameters of the intervertebral discs. Thus, the main task is to obtain accurate goniometric measurements for correct recognition of the type of movements and the attribution of acoustic signals to the values of synovial fluid parameters and the degree of loading.

\section{The system of goniometric control}

The most promising approach to obtaining accurate (up to $0.1^{\circ}$ ) goniometric measurements is the application of an accelerometer method based on the phase-metric principle (figure 2) [15]. Based on the method developed by the authors of this article, the goniometric angle $\Delta \varphi$ is defined by:

$$
\sin \Delta \varphi=\frac{a_{x 2} a_{y 1} / K_{x 2} K_{y 1}-a_{y 2} a_{x 1} / K_{y 2} K_{x 1}}{\left(a_{x 1} / K_{x 1}\right)^{2}+\left(a_{y 1} / K_{y 1}\right)^{2}}
$$


where $a_{x 1}, a_{y 1}, a_{x 2}, a_{y 2}$ are the acceleration values of the first and the second accelerometers in the plane 2D coordinate system; $K_{x 1}, K_{y 1}, K_{x 2}, K_{y 2}$ are transform coefficients of the corresponding accelerometers.

The results of goniometric measurements are compared with the tolerable angles of the individual biomechanical model of the patient. If the measured parameters are close to the boundary values, control signals are generated to determine the type of locomotor system disease (in diagnosis) or to control the actuators (in rehabilitation). The basic values of the amplitude of rotation and inclination of the spine are given in source [16]. The permissible deviation for single vertebrae of an individual n-element angular model is similarly formed.

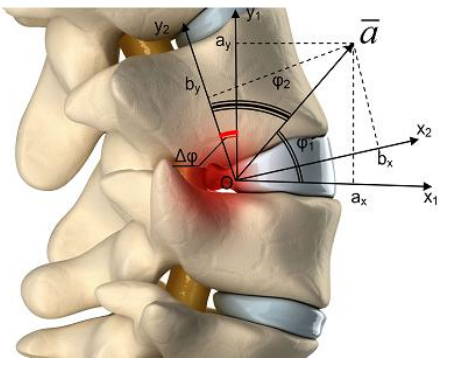

Fig. 2. The accelerometric measurement principle based on the phase-metric principle.

\section{The arthrophonometry in the goniometric control system}

The joint goniometric and arthrophonometric measurements would increase the probability of correct recognition and prediction of the motion type [17], improve the efficiency of system of the diagnostic and rehabilitation of the spine and musculoskeletal apparatus. Atrophonometric data will be allowed to real-time correct of parameters of the coefficient of friction data and the thickness of the synovial fluid layer when moving in an individual three-dimensional biomechanical model [4].

The range of recorded acoustic signals is divided into three sub-ranges: from $180 \mathrm{~Hz}$ to $1 \mathrm{kHz}$ - to record the crunch in the spine; from $1 \mathrm{kHz}$ to $2 \mathrm{kHz}$ - to record the imbalance of muscle tone in the region of the vertebrae and assess the degree of development of pathology in the kinematic pairs of the spine; more than $2 \mathrm{kHz}$ - to assess the volume of interarticulate fluid and to identify the formation of hernias and changes in the properties of the cartilaginous tissue.

The registration of acoustic noise has been proposed by the use of piezoelectric transducers, which in practice is the most convenient [18]. The installation of the piezoelectric transducers is carried out along the spine, which is explained by the smallest number of soft tissues and a small distance to the source of the acoustic signal. The filtration of useful acoustic signals and classification is performed on the basis of wavelet filtration (figure 3) and subsequent neural network processing (figure 4).

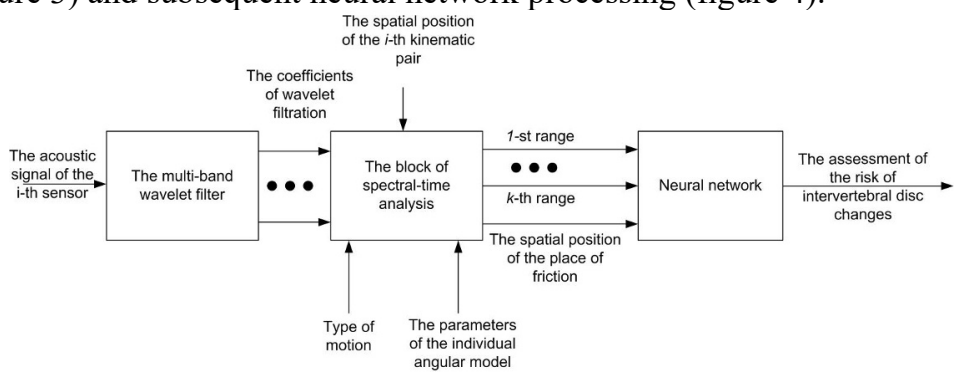

Fig. 3. The algorithm of spectral-temporal processing of acoustic signals. 
The Daubechy filter was chosen as the mother wavelet. The block of spectral-time analysis performs the processing of the obtained values of the coefficients of the filtering unit and, based on additional goniometric information, forms a spatial position vector of the friction zone for further processing by the neural network.

The neural network classifies the degree of friction into three stages for each range and estimates the risk of changes in the intervertebral discs. The neural network has the form of a multilayer recurrent network. The recursion is designed to store for a period of time $t$ information about the emerging noise in the ranges and their total intensity. In the event of a prolonged presence of friction noise or a sufficiently high noise level, a signal of the danger of changing the intervertebral discs is formed. This signal warns of a possible disruption in the functioning of the kinematic pair and can be interpreted on a scale of possible causes during further processing. The registration of background noises in the subscapular region, thoracic and abdominal regions occurs to improve the quality of pathology detection and to reduce the level of interference (interference compensation).

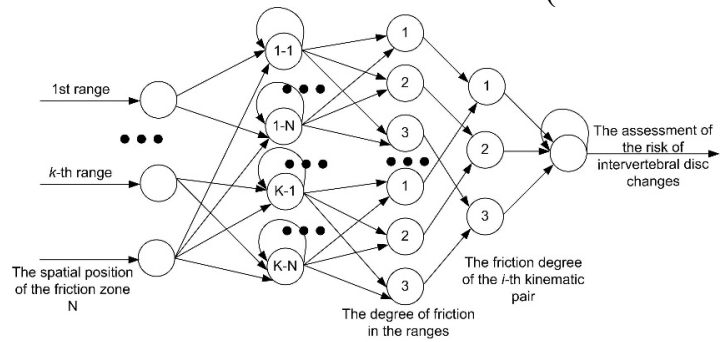

Fig. 4. An example of a neural network for assessing of the risk of changing the intervertebral discs for one kinematic pair.

Verification of the developed algorithms was carried out for a group of 10 patients, for 4 of which showed no abnormal acoustic noise during movement. For 6 patients who have a medical diagnosis - osteochondrosis of the cervical spine, a noise signal of different frequencies was recorded. The data accumulation time $t$ was 5 minutes. As a result of data processing, a signal of danger of pathological changes of the intervertebral discs was obtained for 4 patients with the most severe forms of the diagnosed disease. A false positive (the formation of a signal of danger in healthy patients) was not detection. With an increase in the observation time to 15 minutes, the diagnosis and extent of the disorder were confirmed in all patients.

\section{Conclusion}

Thus, the developed technique for assessing the change in intervertebral discs allows increasing the efficiency of automated systems for diagnosis and rehabilitation of the spine and musculoskeletal system. An improvement of the detection characteristics is possible when correcting the individual biomechanical model and better learning of the neural network.

It should be noted that jointly with the work of the neural network for processing goniometric measurements (described in [12]) and the algorithm for processing acoustic signals, it is possible to estimate the degree of disruption of the functioning of the motor zones of the cerebral cortex, the relationship of which is described in [20].

\section{Acknowledgement}

The work was carried out as part of the research on the project, support by Russian Foundation of Basic Researches, grant No. 16-08-00992_a. 


\section{References}

1. Voznesenskaya T G 2006 Consilium Medicum 8 (2) 75-9

2. Doronin B M and Doronina O B 2010 Neurology, neuropsychiatry, psychosomatics. 20104 24-8.

3. Pezhovych S, Chotek S, Filipyak J, Begzhinsky R, Yarmundovich V and Charek I 2003 Rus. J. of Biomechanics 7 (3) 52-60

4. Pinchuk L S, Chernyakova Yu M and Goldede VA 2008 Friction and wear 29 (3) 293305

5. Yezhov M Yu, Berendeev N N and Petrov SV 2013 Fundamental research 7-3 550-4

6. Tuktamyshev V S and Bezmaternykh V V 2014 Modern problems of sci. and edu. 3 704-9

7. Shilko S V and Ermakov S F 2008 Rus. J. of Biomechanics 12 (2) 31-40

8. Suslov A A, Ermakov S F, Beletsky A V, Shilko S V and Nikolaev V I 2008 Rus. J. of Biomechanics. 12 (4) 33-9

9. Vengerova ]N N and Solovyova I O 2008 Health - the basis of human potential: problems and ways to solve them 3 (1) 45-7

10. Sobolev V I, Popov M N and Anyukhina A V 2017 Problems of Modern Pedagog. Edu. 2017 54-1 227-38

11. Maksimova Yu A 2012 Pedagogy, psychology and medico-biological problems of physic. edu. and sport 12 75-9

12. Dorofeev N, Grecheneva A, Kuzichkin O and Romanov R 2017 17th Int. Multidiscip. Sci. Geocon., SGEM 2017 (Albena; Bulgaria; 29 June 2017 until 5 July 2017) 17 (61) 1161-8

13. Babchina P I, Petrova E M and Chesnokov I V 2017 Innovative materials and technol. in design. Materials of the III All-Rus. Sci. and Tech. Con. P. 37-45

14. Udartsev E Yu, Chantsev A V and Raspopova E A 2009 Travmatology and orthopedics of Rus. 3 (53) 20-7.

15. Grecheneva A V, Dorofeev N V, Kuzichkin O R and Konstantinov I S 2017 Proc. Comp. Sci. $103260-5$

16. Dorofeev N., Podmasteriev K., Kuzichkin O and Grecheneva A 2017 CEUR Workshop Proc. 1839 41-51

17. Zubareva E A 2011 Omsk Scientific Bulletin 1 (104) 165-9

18. Grecheneva A V, Dorofeev N V and Kuzichkin O R 2017 Fund. and Appl. Problems of Engin. and Tech. 5 (325) 163-71

19. Rakhmilevich A B, Chantsev A V, Raspopova E A and Kolomiets A A 2012 Genius of orthopedics 2 102-5

20. Efimov A P 2012 Traumatology and orthopedics of Rus. 1 (63) 60-5 